\title{
Simulation Model of Intravascular Ultrasound Images
}

\author{
Misael Dario Rosales Ramírez ${ }^{1,2}$, Petia Radeva Ivanova ${ }^{2}$, Josepa Mauri $^{3}$, and \\ Oriol Pujol ${ }^{2}$ \\ 1 Laboratorio de Física Aplicada, Universidad de los Andes, Mérida 5133, Venezuela, \\ 2 Centre de Visió per Computador, 08193 Bellaterra, Barcelona, Spain \\ [misael, petia, oriol] @cvc.uab.es \\ 3 Hospital Universitari Germans Trias y Pujol, Badalona, Spain \\ jmauri@ms.hugtip.scs.es
}

\begin{abstract}
The extraction of quantitative information through Intravascular Ultrasound (IVUS) images is a very important goal for the diagnostic and the therapy in atherosclerotic vessels. The correct interpretation highly depends on what gray level values of the image mean, i.e understanding of IVUS image formation. In this project, we propose a simple physical model for simulating IVUS images, based on a discrete representation of the tissue by individual scatterers elements with given spatial distribution and Backscattering Cross Section. This simulation allows studying the significance and the relation between different tissues and the image. Our model allows to study the physics parameters for the IVUS image generation in order to help to the best interpretation (the study of the visibility and robust discrimination of the different structures) as well as to allow creating image data bases to be used during validation of image processing techniques.
\end{abstract}

\section{Introduction}

The introduction of the IntraVascular UltraSound (IVUS) [12] as an exploratory technique has made a significant change to the understanding of the arterial diseases and individual patterns of diseases in the coronary arteries. Huge amount of data and difficult interpretation pushed developing of image processing techniques, border segmentation, plaque characterization, multi-modal fusion [3, etc. The question is how to develop robust algorithms that can solve these problems analyzing the artifacts with their multiple appearance in IVUS images. Having a complete set of patient data to present all variance of artifacts appearance in images would mean to dispose of a huge amount of patient cases. A more efficient solution is to develop a simulation model for IVUS data construction so that synthetic data are available in order to "train" image processing techniques. Image study based on modelling the image generation process is a relatively new field, there is a different works [4.56] oriented to the simulation of conventional ultrasound images, based on the interaction of sound waves and the biological tissue. Nevertheless, there is no recent investigation about the basic physical principles, as well as the protocols of image processing to obtain intravascular ultrasound images. In this way, different appearance of artifacts can be designed 
to assure the robust performance of image processing techniques. Differences in IVUS data are caused not only by different morphological structures of vessels but also by different parameters that influence the formation of IVUS images. The images depend on the IVUS apparatus calibration as well as interventional devices, small differences in parameters can lead to different grey-level appearance that can be interpreted in a different way by the physicians. Having a simulation model for IVUS data can help to the training of medical staff as well as can have an important role in designing and testing new interventional devices. At the end, being aware which parameters and in which grade influence to image formation is of unquestionable importance for all persons involved in comprehension of IVUS data and taking final decision for diagnosis and intervention of vessel lesions. The article has been organized as follow: Section 2 we discuss a simple simulation model for formation of 2D IVUS data that explains the complete process of data generation as a result of the interaction between ultrasound signals and vessel morphological structures, section 3 the validation and results are discussed and the conclusions are presented in section 4 .

\subsection{Formal Definition of the Image Model}

IVUS images can be obtained in a simulated form, from a simple physical model based on the transmission and reception of high frequency sound waves, when these radially penetrate a simulated arterial structure (Fig. 11 (a)). Let us consider a ultrasound pulse $P_{o}$ that is emitted at time $t_{o}$ with speed $c$ from the point with coordinates $\left(r_{o}, \theta_{o}, z_{o}\right)$ (Fig. 1 (b)), and that interacts with the scatterer located at the position, $(R, \Theta, Z)$ with the spatial distribution of the differential backscattering cross-section, $\sigma(R, \Theta, Z)$. The reflected pulse $P_{i}$ for the i-th scatterer is an exact replica [7] of the transmitted sound pulse $P_{o}$ that will return to the point $\left(r_{o}, \theta_{o}, z_{o}\right)$ at time $\left(t_{i}-t_{o}\right)$ and will be out of phase temporarily with respect to the pulse $P_{o}$ by time difference $\delta=t_{i}-t_{o}$ between the emitted pulse at $t_{i}$ and the received pulse at $t_{o}$. The time delay $\delta$ is given by $\delta=2|R| / c$. Assuming the Born approximation 89, the reflected signal $S(t, \tau)$ for a finite set of $N$ reflecting scatterers with coordinates $(R, \Theta, Z)$ and spatial distribution of the differential backscattering cross-section $\sigma(R, \Theta, Z)$ is given by:

$$
S(R, \Theta, Z, t, \tau)=\sum_{i=1}^{N} \sigma_{i}(R, \Theta, Z) \zeta_{i}(t, \tau)
$$

where $N$ is the number of scatterers, $\sigma_{i}(R, \Theta, Z)$ is the spatial distribution of the Differential Backscattering Cross-section (DBC) of the i-th scatterer located in position $(R, \Theta, Z), \zeta_{i}(t, \tau)$ is the transducer impulse function and $\tau$ is the delay time which leads to constructive and destructive contributions to the received signal. We consider a planar transducer that is mounted inside an infinite baffle, so that the ultrasound is only radiated in the forward direction 10 11. The far field circular transducer pressure $P(r, \theta, t)$ can be written as:

$$
P(r, \theta, t)=j \frac{\rho_{o} c k a^{2} v_{o}}{2 r}\left[\frac{2 J_{1}(k a \sin (\theta))}{k a \sin (\theta)}\right] \exp (j(w t-k r))
$$




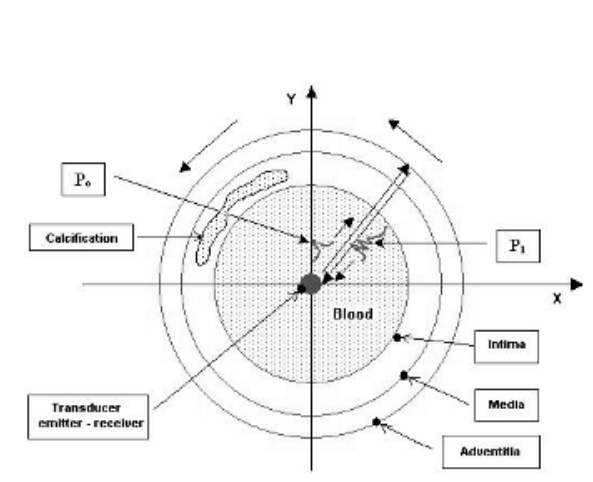

(a)

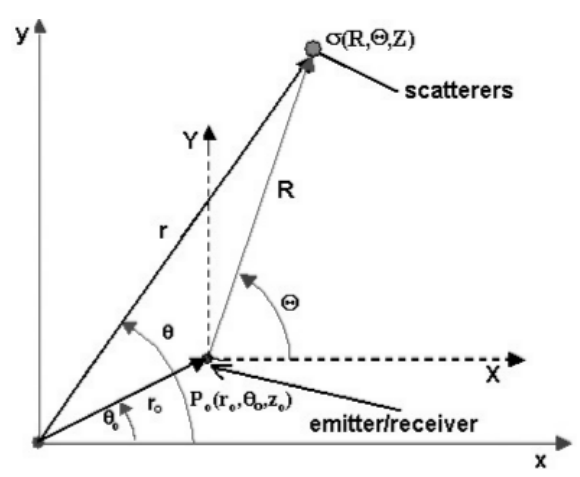

(b)

Fig. 1. The disposition (a) of the simulated arterial structures and calcification. The coordinates system (b) used in the model.

where $t$ is the time, $\rho_{o}$ is the medium propagation density, $c$ is the sound speed for biological tissue, $v_{o}$ is the radial speed at a point of the transducer surface, $a$ is the transducer radius, $\vec{k}$ is the propagation vector, defined as $k=|\vec{k}|=2 \pi / \lambda$, $\lambda$ the ultrasound wave length, defined as $\lambda=c / f o, f_{o}$ ultrasound frequency, $\omega=2 \pi f_{o}$ and $J_{1}(x)$ is the first class Bessel function. The impulse function $\zeta(t, \delta)$ is generally approximated by a Gaussian which envelopes the intensity distribution and is given by: $\zeta(t, \delta)=I(r, \theta, t) \exp \left(-(t-\delta)^{2} / 2 \sigma^{2}\right)$, where $\sigma$ is the pulse standard deviation. We consider that the beam is collimated by $\theta=$ $\theta_{a}$. Hence Eq. (II) in the transducer coordinate system is based on a discrete representation of the tissue of individual scatterer elements with given position and $D B C$ with respect to the transducer coordinates given by:

$$
S(R, \Theta, Z, t, \delta)=C_{o} \sum_{i=1}^{N} \frac{\sigma_{i}(R, \Theta, Z)}{\left|R_{i}\right|} \zeta(t, \delta)
$$

If we consider only the axial intensity contributions, $C_{o}$ can be written as [10]: $C_{o}=\rho_{o} c k^{2} v_{o}^{2} A / 8 \pi$, where $A$ is the transducer area.

The beam ultrasound intensity, as a function of the penetration depth and the ultrasound frequency, is given by [1011]12]: $I(r)=I_{o} \exp \left(-\alpha\left(N_{\theta}\right) r f\right)$ where $I_{o}$ is the beam intensity at $r=0$ and the coefficient, $\alpha$ give the rate of diminution of average power with respect to the distance along a transmission path [16]. It is composed of two parts, one (absorption) proportional to the frequency, the other (scattering) dependent on the ratio of grain, particle size or the scatterer number $N_{\theta}$ located along the ultrasound beam path.

\subsection{Determining the Scatterer Number of Arterial Structures}

The Red Blood Cells (RBCs) number swept by the ultrasound beam (Fig. 2) can be estimated taking into account the plastic sheathing dimensions of the 


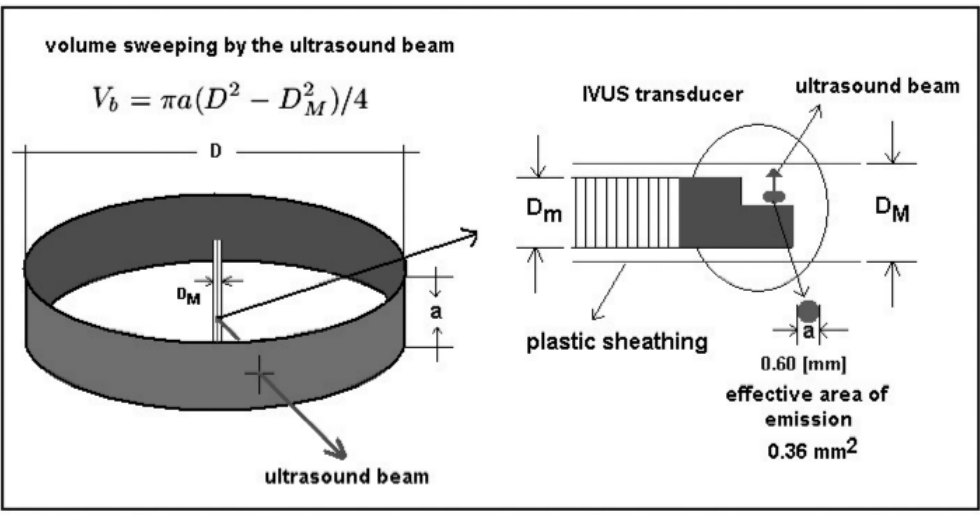

Fig. 2. The scatterers volume for each arterial structure can be calculated taken into account the total volume $V_{b}$ swept by the ultrasound beam.

transducer and the typical arterial lumen diameter. The scatterer number contained in a sweeping beam volume is given by the difference between the sweeping lumen arterial volume, $V_{a}$ and the plastic sheathing transducer volume, $V_{t}$. $V_{b}=V_{a}-V_{t}=\pi a\left(D^{2}-D_{M}^{2}\right) / 4$, where $D$ and $D_{M}$ are the arterial lumen and the sheathing transducer exterior diameters respectively, and $a$ is the effective emission diameter of the transducer. The numerical values necessary for the evaluation of the DBC scatterer distribution for the intima, media and adventitia were taken from results of L. T. Perelman et. al [13, that give the typical nuclear cells size $l(\mu m)$ distribution for human cells. The "voxel" number for each layer was computed taken into account the typical dimensions of intima, media and adventitia of a normal artery. The voxel number for the sheathing transducer was calculated taking into account the minimal scatterers able to be observed at maximal resolution when the frequency is fixed at $40 \mathrm{MHz}$, a typical IVUS frequency.

\subsection{Generation of the Simulated Arterial Structure}

Considering the goal of simulating different arterial structures, we can classify them in 3 groups: Tissue structures, non tissue structures and artifacts. The spatial distribution of the scatterer number with a given $\mathrm{DBC}, \sigma(R, \Theta, Z)$ at point $(R, \Theta, Z)$ has the following contributions: $\sigma(R, \Theta, Z)=A(R)+B(R, \Theta, Z)+$ $C(R)$, where $\mathrm{A}(\mathrm{R})$ are the tissue scatterers, these are determined by the contribution of the normal artery structures, corresponding to: lumen, intima, media and adventitia. $B(R, \Theta, Z)$, are non tissue scatterers. These contributions can be originated by structures formed by spatial calcium accumulation, which are characterized because the DBC density is greater than the rest of the arterial structures and $\mathrm{C}(\mathrm{R})$ are the artifacts scatterers. In our model we consider only the artefact caused by the sheathing transducer. 


\subsection{Image Generation Procedures}

The procedures to obtain the final simulated image are as follows: 1) The echoes are obtained by the pivoting transducer (Fig. 3 (a)). Each echo profile is ordered according to the angular position (Fig. 3 (b)). The original image is transformed to a polar form (Fig. 3 (c)). 4) Secondary beams are computed between two original neighbor beam (Fig. 3 (c)) The image is smoothed by a $2 \times 2$ median filter. 6) The image is again transformed to cartesian form. As result of this transformation a significant number of pixels will be empty. 7) The empty pixels are filled in a recursive way form, using for this an average of the eight nearest neighbors. 8) An image reference reticle is added and a gaussian filter is applied.

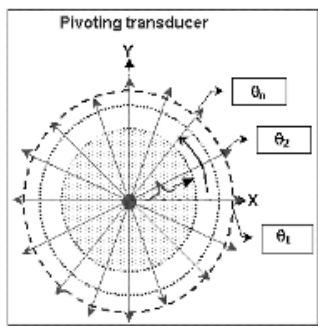

(a)

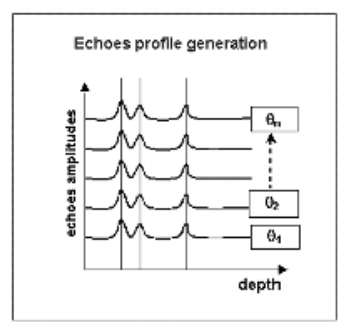

(b)

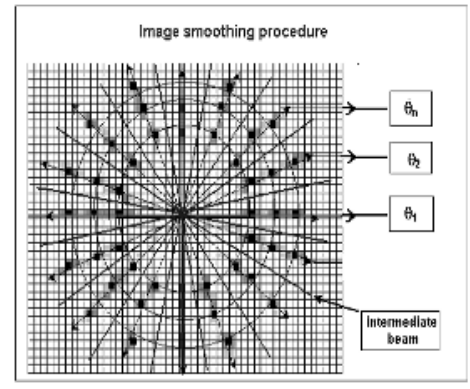

(c)

Fig. 3. The transducer emits from the artery center (a), echoes profile (b), the final IVUS image is smoothed (c).

\section{Validation of the Image Simulation Model}

Once defined the generic basic model of IVUS image formation, we need to compare it to real images contrasting the expert opinion to test its use. In order to compare the real and simulated IVUS images, we have generated 20 synthetic images with morphological structures corresponding to the structures of a set of real images. We have used a real IVUS image with manually delimited lumen, intima, and adventitia to obtain the average radius location, $\bar{R}_{k}$ for each arterial structure. We applied the optimal frequency of $46 \mathrm{MHz}$ and attenuation coefficient 0.8 [dB/MHZ cm] obtained by cross validation method [14. Fig. 4 shows an IVUS real image of right coronary artery (a) obtained by a $40 \mathrm{MHz}$ Boston Sci. equipment 15 and simulated (c) cartesian IVUS images and the corresponding real (b) and simulated (d) polar transformations. The global appearance of each image region (lumen, intima, media and adventitia) and their corresponding interface transitions (lumen/intima, intima/media and media/adventitia) are visually well contrasted, compared to the real image. We can see a good gray 


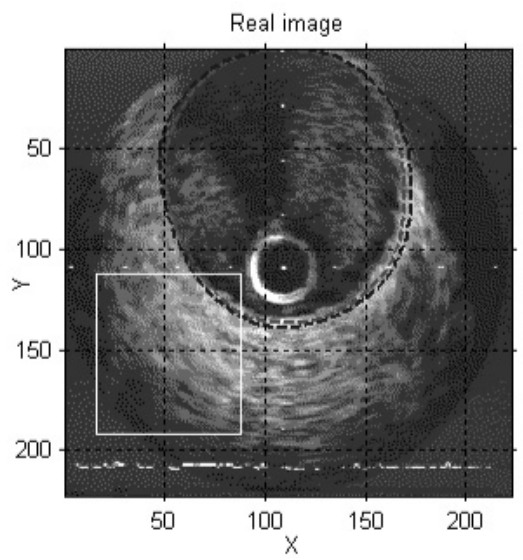

(a)

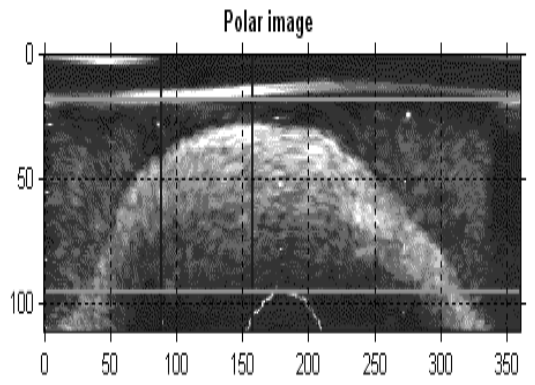

(c)

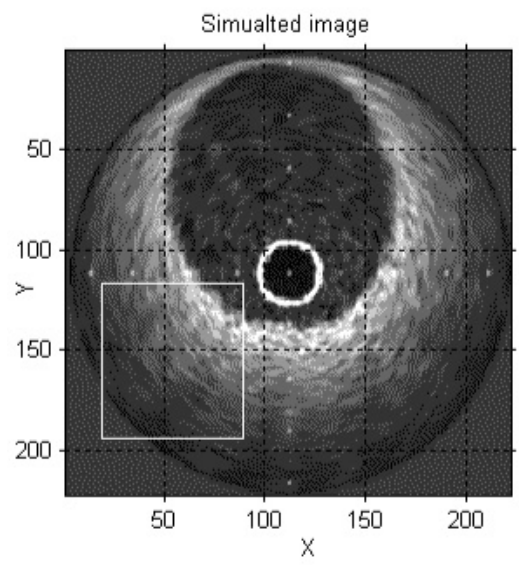

(b)

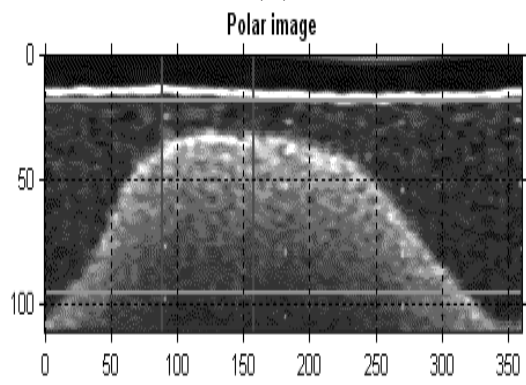

(d)

Fig. 4. Real (a) and simulated (b) IVUS images segmentation and their corresponding real (b) and simulated (d) polar transformation. ROIs are given as squares. Manual segmentation of the vessel is given in (a).

level distribution and a soft grey level decay from the center to the peripheries of the IVUS image, produced by the inverse relation between the ultrasound intensity and the penetration depth. Real IVUS images and their corresponding ROIs were selected, the spatial boundaries of the morphological structures of the real data are kept in the synthetic data. Fig. 5 (a) shows 10 real and their corresponding simulated (b) synthetic images. Figure 5 (c) shows the simulated vs real gray level correlation for the polar ROI's images selected as shown in (Fig. 4 (c) and (d)). The linear correlation coefficients show a good gray levels correspondence, being these $\mathrm{m}=0.90$ and $\mathrm{b}=1.42$. The best correspondence is located by low gray levels (20 to 40 gray levels), lumen scatterers, lumen/intima transition, and adventitia. The transition of intima/media and media/adventitia (45 to 60 gray levels) indicate a gradual dispersion. The contrast to noise ratio CNRS as figure of similarity for each arterial validated region is shown in (Fig. 


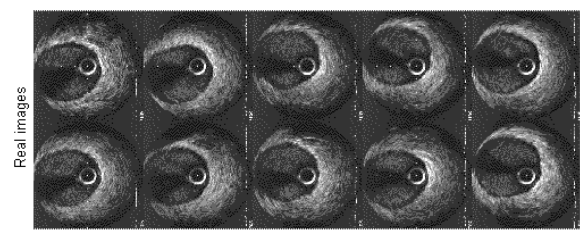

(a)

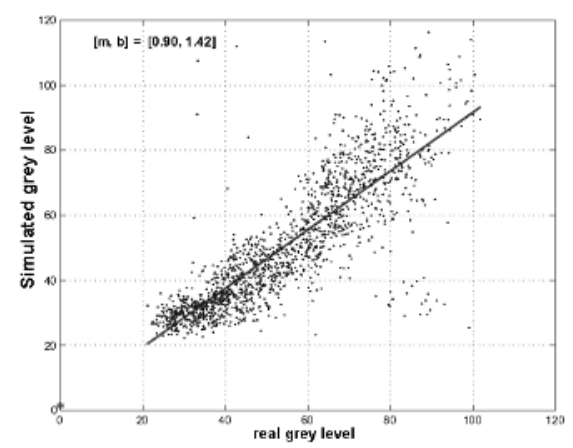

(c)

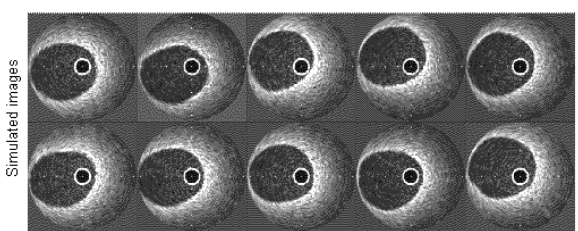

(b)

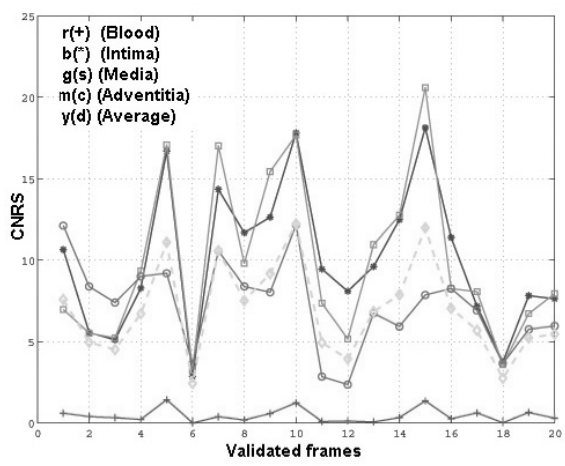

(d)

Fig. 5. 10 original IVUS images (a) and the corresponding simulated (b) images. Simulated vs real grey level ROIs values (c) and its CNRS values (c)

[5(d)). The CNRS average presents a significative uniformity values, $\mu=6.89$ and $\sigma=2.88$, for all validated frames.

\section{Conclusions}

Although IVUS is continuously gaining its use in practice due to its multiple clinical advantages, the technical process of IVUS image generation is not known by doctors and researchers developing IVUS image analysis. This fact leads to a simplified use, analysis and interpretation of IVUS images based only on the grey level values of image pixels. In this paper we discuss a basic physical model to generate synthetic 2D IVUS images. The model has different utilities: Firstly, expert medical doctors can generate simulated IVUS images, in order to observe different arterial structures of clinical interest and their grey level distribution in real images. Secondly, medical doctors can use our model to learn and to compare the influence of different physical parameters in the IVUS image formation, for example: the ultrasound frequency, the attenuation coefficient, the beam number influence, and the artifact generations. The obtained results of the validation of our model illustrate a good approximation to the image formation process. The 2D IVUS images show a good correspondence between the arterial structures that generate the image structures and their gray level values. Interested readers are invited to check the generation model in (http://www.cvc.uab.es/ misael). 


\section{References}

1. Yock P., Linker D., Saether O., et al., Intravascular two dimensional catheter ultrasound, Initial clinical studies, abstracted, Circulations, No. 78 (suppl II): II-21, 1988.

2. Graham S., Brands D., Sheehan H., et al., Assesment of arterial wall morphology using intravascular ultrasound in vitro and in patient, Circulations, (Suppl II): II-56, 1989.

3. Berry E., and et al, Intravascular ultrasound-guided interventions in coronary artery disease, Tech. Rep., Healt Technology Assesment, NHS R D HTA Programme. A systemac literature review, with decisions-analytic modelling, of outcomes and cot-effectiveness, 2000.

4. Jensen J., A Model for the Propagation and Scattering of Ultrasound in Tissue, J. Acoust. Soc. Am, 1991, 89, 182-191.

5. Jensen J., A Program for Simulating Ultrasound Systems,Paper presented at the 10th Nordic-Baltic Conference on Biomedical Imaging Published in Medical and Biological Engineering and Computing, 1996, Part 1, Supplement 1, 351-353.

6. Trobaugh J., An Image Model for Ultrasound Incorporating Surface Shape and Microstructure and Characteristics of Imaging System, Washington University, Sever Institute of Technology, Departmet of Electrical Ingeniering, 2000, Doctoral thesis.

7. Verhoef, W.A, Cloostermans, M.J, and Thijssen, J.M, The Impulse Response of a Focused Source With an Arbitrary Axisymmetric Surface Velocity Distribution, Journal Acoustic Society American, 1984, 75", 1717-1721.

8. Fontaine I., Bertrand M, Cloutier G., A system-based approach to modelling the ultrasound signal backscattered by red blood cells,Biophysical Journal, Vol. 77, pp. 2387-2399, 1999

9. Fan L., Herrington D., Santiago P., Simulation of b-mode ultrasound to determine features of vessel for image analysis, Computers in Cardiology, Vol. 25, pp. 165168,1998

10. Cheeke D., Fundamentals and Aplications of Ultrasonic Waves, CRC PRESS, 2002.

11. Kinsler L. , Fundamentos de acústica, LIMUSA, Noriega Editores, 1995.

12. Zagzebski J., Essential of Ultrasound Physics, Mosby A. hardcourt Healt Sciences Company, 1996.

13. Perelman L., and et al, Observation of periodic fine structure in reflectance from biological tissue: A new technique for measuring nuclear size distribution, Physical Review Letters, Vol. 80, No. 3, pp. 627-630, January 1998.

14. Rosales M., Radeva P., A basic model for IVUS image simulation in Handbook of Medical Imaging, Kluwer Academic/Plenium Publishers, 233 Spring Street, New York, NY 10013, USA, (in press), 2004.

15. Boston Scientific Corporation, Scimed division, The ABCs of IVUS, 1998.

16. Arendt Jesen J., Linear Descripcion of Ultrasound Imaging System, Notes for the international Summer School on Advanced Ultrasound Imaging, Tecnical University of Denamark, 2001. 